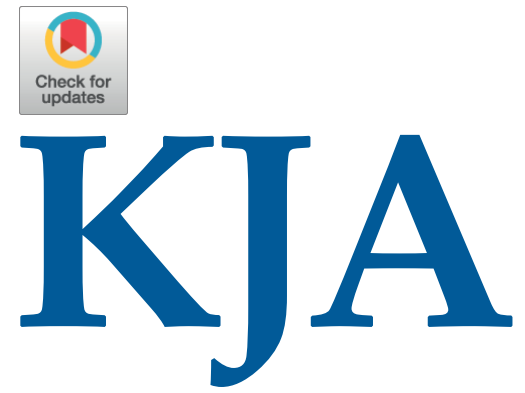

Korean Journal of Anesthesiology

\section{Letter to the Editor}

Korean J Anesthesiol 2021;74(4):366-367

https://doi.org/10.4097/kja.20687

pISSN 2005-6419 • eISSN 2005-7563

Received: December 29, 2020

Revised: January 1, 2021

Accepted: January 4, 2021

Corresponding author:

George Zhong, FANZCA, MBBS(Hons),

BSc(Medal), GradCert(Biostats)

Department of Anesthesia, Concord

Repatriation General Hospital, 1A Hospital

Road Concord NSW 2139, Australia

Tel: +61-2 87593223

Fax: +61-4 24265325

Email: drgzhong@gmail.com

ORCID: https://orcid.org/0000-0003-0286-4606

\title{
Propofol extravasation pain masked by lignocaine premedication
}

\author{
George Zhong $^{1,2}$, Shun Hin Kenjo Ho ${ }^{1,3}$, Alpha Mang Sze Tung ${ }^{1,4}$ \\ ${ }^{1}$ Department of Anesthesia, Auburn Hospital, ${ }^{2}$ Department of Anesthesia, Concord Repatriation \\ General Hospital, ${ }^{3}$ School of Medicine, University of Sydney, ${ }^{4}$ School of Medicine, University of \\ Notre Dame, Sydney, Australia
}

Propofol injection is associated with pain in approximately $60 \%$ of untreated cases [1]. The pain is usually sharp or burning and can be severe. Several pharmacological treatments have been shown to reduce pain associated with propofol injections [2]. Of these, premedication with lignocaine is the most frequently used and the most reported in the literature. Here, we report a case of the complete masking of pain associated with propofol extravasation in an awake patient following lignocaine premedication, which markedly delayed its diagnosis. Informed consent was obtained from the patient for this publication.

A 50-year-old lady (weight $116 \mathrm{~kg}$, body mass index $43 \mathrm{~kg} / \mathrm{m}^{2}$ ) with known difficult venous access presented for surveillance gastroscopy and colonoscopy under sedation. After multiple failed attempts, a cannula was inserted into the antecubital fossa vein, through which fentanyl $(50 \mu \mathrm{g})$ and midazolam $(1 \mathrm{mg})$ were administered. Lignocaine (100 mg, $10 \mathrm{ml}$ ) was slowly administered through the cannula before a target-controlled infusion of propofol (Provive ${ }^{\circledR}$ MCT-LCT 1\%, Baxter Healthcare, Australia). Despite the injection of more than $300 \mathrm{mg}$ of propofol within $2 \mathrm{~min}$, the patient had no evidence of sedation. A presumptive diagnosis of propofol extravasation was made, and the infusion was discontinued. The patient did not report any discomfort, and the antecubital fossa cannula site remained soft and non-tender on examination. A second cannula was inserted in the contralateral arm, and propofol target-controlled infusion was recommended with a rapid clinical response. The remainder of the procedure and sedation were uneventful. The patient was monitored for two hours in the post-anesthesia recovery unit, where mild erythema began to develop around the original antecubital fossa cannula site. The patient also reported experiencing pain during elbow flexion. Her arm remained soft, distal pulses were present, and she was deemed safe for discharge. On a telephone follow-up the next day, the patient reported that the erythema and pain in her arm had completely resolved.

There have been numerous reports of propofol extravasation in the literature, with sequelae ranging from local erythema to tissue necrosis and compartment syndrome [3-5]. In all published cases, propofol extravasation involved patients who were unable to voice pain (anesthetized, sedated, or neonate). Missed extravasation of large volumes of propofol in an awake patient is rare because extravasation pain is usually severe, which leads to early detection and cessation of administration.

In the present case, the detection of propofol extravasation was significantly delayed due to the absence of patient discomfort. Extravasation was only suspected after a relatively large volume $(30 \mathrm{ml})$ of propofol was administered without any observable pharmacodynamic response. The absence of extravasation pain, in this case, was likely due to the anesthetic effect of lignocaine and the location of the cannula. The lignocaine premedication may have also been administered into the subcutaneous tissue in the antecu- 
bital fossa, which readily diffused to nearby nerve fibers, resulting in anesthesia of the region. This effectively masked the discomfort resulting from the direct chemical irritation by propofol on the local nociceptors. Furthermore, the antecubital fossa provided a large potential space that enabled a significant volume (over $40 \mathrm{ml}$ in total) of extravasate to accumulate, masking the discomfort that resulted from increased compartmental pressures. A higher than normal dose of lignocaine was used during premedication in this case with the additional aims of facilitating insertion of the gastroscope and reducing cough, which further increased the risk of masking the pain associated with propofol extravasation.

Given the increasing use of propofol-based total intravenous anesthesia, clinicians need to detect extravasation promptly to minimize morbidity. Clinicians need to pay attention to all signs of extravasation, including patient discomfort, elevated injection pressure, changes at the cannula site, and the absence of clinical response, as demonstrated in this case. Antecubital fossa veins should be avoided, as signs of extravasation are harder to detect in them.

To our knowledge, this is the first reported case of lignocaine premedication masking the pain associated with propofol extravasation in an awake patient. Anesthesiologists should be aware of the possibility of painless propofol extravasation, especially after premedication with lignocaine.

\section{Conflicts of Interest}

No potential conflict of interest relevant to this article was reported.

\section{Author Contributions}

George Zhong (Conceptualization; Writing - original draft, review \& editing; Supervision)

Shun Hin Kenjo Ho (Conceptualization; Literature Review)

Alpha Mang Sze Tung (Conceptualization; Writing - review \& editing)

\section{ORCID}

George Zhong, https://orcid.org/0000-0003-0286-4606

Shun Hin Kenjo Ho, https://orcid.org/0000-0002-3271-3203

Alpha Mang Sze Tung, https://orcid.org/0000-0001-9013-9150

\section{References}

1. Picard P, Tramèr MR. Prevention of pain on injection with propofol: a quantitative systematic review. Anesth Analg 2000; 90: 963-9.

2. Jalota L, Kalira V, George E, Shi YY, Hornuss C, Radke O, et al. Prevention of pain on injection of propofol: systematic review and meta-analysis. BMJ 2011; 342: d1110.

3. Huijbers EJ, Baars JW, Schutte PF, Schellens JH, Beijnen JH. Propofol extravasation in a breast cancer patient. J Oncol Pharm Pract 2008; 14: 195-8.

4. Tokumine J, Sugahara K, Tomori T, Nagasawa Y, Takaesu Y, Hokama A. Tissue necrosis caused by extravasated propofol. J Anesth 2002; 16: 358-9.

5. Kalraiya AJ, Madanipour S, Colaco H, Cobiella C. Propofol extravasation: a rare cause of compartment syndrome. BMJ Case Rep 2015; 2015: bcr2015209360. 